\title{
LATTICES WITH THE ALEXANDROV PROPERTIES
}

\author{
ALBERT GORELISHVILI
}

(Communicated by Andrew M. Bruckner)

\begin{abstract}
By an Alexandrov lattice we mean a $\delta$ normal lattice $\mathscr{L}$ of subsets of an abstract set $X$, such that the set of $\mathscr{L}$-regular countably additive bounded measures, denoted by $\operatorname{MR}(\sigma, \mathscr{L})$, is sequentially closed in the set of $\mathscr{L}$. regular finitely additive bounded measures on the algebra generated by $\mathscr{L}$, i.e., if $\mu_{n} \in \operatorname{MR}(\sigma, \mathscr{L})$ and $\mu_{n} \rightarrow \mu$ (weakly) then $\mu \in \operatorname{MR}(\sigma, \mathscr{L})$.

For a pair of lattices $\mathscr{L}_{1} \subset \mathscr{L}_{2}$ in $X$ sufficient conditions are indicated to determine when $\mathscr{L}_{1}$ Alexandrov implies that $\mathscr{L}_{2}$ is also Alexandrov and vice versa. The extension of this situation is given where $T: X \rightarrow Y, \mathscr{L}_{1}$ and $\mathscr{L}_{2}$ are lattices of subsets of $X$ of $Y$ respectively, and $T$ is $\mathscr{L}_{1}-\mathscr{L}_{2}$ continuous.
\end{abstract}

\section{INTRODUCTION}

Alexandrov's fundamental theorem [1] states that if $\mathscr{L}$ is a $\delta$ normal lattice of subsets of a set $X$ and if $\mathscr{L}$ is also complement generated (completely normal), then $\mu_{n} \in \operatorname{MR}(\sigma, \mathscr{L})$ and $\mu_{n} \stackrel{w}{\rightarrow} \mu$, where $\mu \in \operatorname{MR}(\mathscr{L})$, imply $\mu \in \operatorname{MR}(\sigma, \mathscr{L})$ (see below for definitions). A different proof of this appears in [4] as well as in [6] where it is proved for the case of the lattice of zero sets of a completely regular topological space.

Our aim here is to consider other lattices that have this fundamental Alexandrov property; such lattices will be called Alexandrov lattices. In particular, we will consider the situation of two lattices $\mathscr{L}_{1}, \mathscr{L}_{2}$ of subsets of $X$ such that $\mathscr{L}_{1} \subset \mathscr{L}_{2}$ and will determine conditions under which $\mathscr{L}_{1}$ is Alexandrov implies $\mathscr{L}_{2}$ is Alexandrov and conversely. We will also consider the case of two sets $X$ and $Y$ and appropriate lattice mappings between them and subsequently adjoint mappings in order to determine further Alexandrov lattices. Our terminology and notation are consistent with $[1,2]$. For the reader's convenience, we will repeat several special notations and the lattice terminology needed in this paper.

\section{Definitions AND NOtATIONS}

Let $X$ be a set and $\mathscr{L}$ be any lattice of subsets of $X$. We shall always assume that $\phi, X \in \mathscr{L}$. We use the following notation: $R$ for the real numbers, $x$ for the general element of $X, \mathfrak{A}(\mathscr{L})$ for the smallest algebra containing $\mathscr{L}, \sigma(\mathscr{L})$ for the smallest $\sigma$-algebra containing $\mathscr{L}, \delta(\mathscr{L})$ for the smallest

Received by the editors October 29, 1990.

1991 Mathematics Subject Classification. Primary 28A32, 46E27, 28C15, 46G12. 
$\delta$-lattice containing $\mathscr{L}, \tau(\mathscr{L})$ for the smallest $\tau$-lattice containing $\mathscr{L}$, and $s(\mathscr{L})$ for the Souslin sets determined by $\mathscr{L} . \delta(\mathscr{L})$ is the set of all $\bigcap L_{i}$ with $L_{i} \in \mathscr{L}, i=1,2, \ldots$. If $\mathscr{L}$ is closed under countable intersections, then $\mathscr{L}$ is $\delta$ or a $\delta$-lattice. $\mathscr{L}$ is complemented if $A \in \mathscr{L}$ implies $A^{\prime} \in \mathscr{L}$ where $A^{\prime}=X-A . \mathscr{L}^{\prime}$ is the class of all complements of $\mathscr{L}$-sets, i.e., $\mathscr{L}^{\prime}=$ $\left\{L^{\prime}: L \in \mathscr{L}\right\} . \mathscr{L}$ is complement generated if $A \in \mathscr{L}$ implies $A=\bigcap A_{i}^{\prime}$ where $A_{i} \in \mathscr{L}, i=1,2, \ldots$.

$\mathscr{L}$ is disjunctive if for any $A \in \mathscr{L}$ and $x \notin A$ there exists a $B \in \mathscr{L}$ such that $x \in B$ and $A \cap B=\varnothing$. $\mathscr{L}$ is countably paracompact (c.p.) if $A_{n} \in \mathscr{L}$ and $A_{n} \downarrow \phi$ imply there exist $B_{n} \in \mathscr{L}$ such that $A_{n} \subset B_{n}^{\prime}$ and $B_{n}^{\prime} \downarrow \phi$.

Let $\mathscr{L}_{1}$ and $\mathscr{L}_{2}$ be two lattices of subsets of $X$ and $\mathscr{L}_{1} \subset \mathscr{L}_{2}$. $\mathscr{L}_{1}$ semiseparates (s.s.) $\mathscr{L}_{2}$ if for every $A \in \mathscr{L}_{1}$ and $B \in \mathscr{L}_{2}, A \cap B=\varnothing$ implies there exists a $C \in \mathscr{L}_{1}$ such that $B \subset C$ and $A \cap C=\varnothing ; \mathscr{L}_{1}$ separates $\mathscr{L}_{2}$ if for all $A, B \in \mathscr{L}_{2}, A \cap B=\varnothing$ implies there exist $C, D \in \mathscr{L}_{1}$ such that $A \subset C$, $B \subset D, C \cap D=\varnothing . \mathscr{L}_{2}$ is $\mathscr{L}_{1}$ countably paracompact if for every $A_{n} \in \mathscr{L}_{2}$, $A_{n} \downarrow \phi$ imply there exist $B_{n} \in \mathscr{L}_{1}$ such that $A_{n} \subset B_{n}^{\prime}$ and $B_{n}^{\prime} \downarrow \phi . \mathscr{L}_{2}$ is $\mathscr{L}_{1}$ countably bounded (c.b.) if for all $A_{n} \in \mathscr{L}_{2}, A_{n} \downarrow \phi$ imply there exist $B_{n} \in \mathscr{L}_{1}$ such that $A_{n} \subset B_{n}$ and $B_{n} \downarrow \phi$.

A function $f: X \rightarrow R \cup\{ \pm \infty\}$ is $\mathscr{L}_{1}$-continuous if $f^{-1}(C) \in \mathscr{L}$ for every closed set $C \subset R \cup\{ \pm \infty\}$. The algebra of all $\mathscr{L}$ continuous function is denoted by $C(\mathscr{L})$; the algebra of bounded continuous functions is denoted by $C_{b}(\mathscr{L})$. The set whose general element is a zero set of $\mathscr{L}$ is denoted by $\mathscr{Z}(\mathscr{L})$; $Z \in \mathscr{Z}(\mathscr{L})$ iff $Z=f^{-1}(0)$ for some $\mathscr{L}$-continuous function $f$. A measure on $\mathfrak{A}(\mathscr{L})$ is a finitely additive bounded real-valued set function. $M(\mathscr{L})$ denotes the set of all measures on $\mathfrak{A}(\mathscr{L})$. A measure $\mu$ is said to be $\sigma$-smooth on $\mathscr{L}$ if for every $A_{n} \in \mathscr{L}, A_{n} \downarrow \phi$ imply $\mu\left(A_{n}\right) \rightarrow 0$. A measure $\mu \in \mathrm{M}(\mathscr{L})$ is said to be $\mathscr{L}$-regular if for every $A \in \mathfrak{A}(\mathscr{L})$ and every $\varepsilon>0$, there exists an $L \in \mathscr{L}$ such that $L \subset A$ and $|\mu(A)-\mu(L)|<\varepsilon$. The set whose general element is an element of $\mathscr{L}$, which is $\sigma$-smooth on $\mathscr{L}$, is denoted by $\mathbf{M}(\sigma, \mathscr{L})$. Moreover, we use the notation $\operatorname{MR}(\sigma, \mathscr{L})=\operatorname{MR}(\mathscr{L}) \cap M(\sigma, \mathscr{L})$. The set of all measures such that $\mu(A)=\{0,1\}$ for every $A \in \mathfrak{A}(\mathscr{L})$ and $\mu(X)=1$ is denoted by $\mathrm{I}(\mathscr{L})$. The set of all $\{0,1\}$-valued $\mathscr{L}$-regular measures is denoted by $\operatorname{IR}(\mathscr{L})$, i.e., $\operatorname{IR}(\mathscr{L})=\mathrm{I}(\mathscr{L}) \cap \operatorname{MR}(\mathscr{L})$.

For the restriction of $\nu \in \operatorname{MR}\left(\mathscr{L}_{2}\right)$ to $\mathfrak{A}\left(\mathscr{L}_{1}\right)$, we adopt the notation $\left.\nu\right|_{\mathscr{L}_{1}}$, or simply, $\nu \mid$. Note that if $\nu \in \operatorname{MR}\left(\mathscr{L}_{2}\right)$ and if $\mathscr{L}_{1}$ s.s. $\mathscr{L}_{2}$, then $\nu \mid \in \operatorname{MR}\left(\mathscr{L}_{1}\right)$.

We conclude this section with the following general extension theorem and state for reference Alexandrov's Representation Theorem (A.R.T).

Theorem 2.1 [4]. Let $\mathscr{L}_{1}$ and $\mathscr{L}_{2}, \mathscr{L}_{1} \subset \mathscr{L}_{2}$, be two lattices of subsets of $X$. Then, any measure $\mu \in \operatorname{MR}\left(\mathscr{L}_{1}\right)$ can be extended to a $\nu, \nu \in \operatorname{MR}\left(\mathscr{L}_{2}\right)$, and if $\mathscr{L}_{1}$ separates $\mathscr{L}_{2}$ then $\nu$ is unique. If $\mu \in \operatorname{MR}\left(\sigma, \mathscr{L}_{1}\right)$ and $\mathscr{L}_{2}$ is $\mathscr{L}_{1}$ countably paracompact or countably bounded, then $\nu \in \operatorname{MR}\left(\sigma, \mathscr{L}_{2}\right)$.

Theorem (Alexandrov) [1b]. Let $\mathscr{L}$ be a $\delta$ normal lattice. Then the conjugate space $C_{b}(\mathscr{L})^{\prime}$ of $C_{b}(\mathscr{L})$ is $\operatorname{MR}(\mathscr{L})$. In more details: To every bounded linear functional $\Phi$ there corresponds a unique $\mu \in \operatorname{MR}(\mathscr{L})$ such that $\Phi(f)=\int f d \mu$ with $\|\Phi\|=|\mu|$. The positive and negative parts of $\Phi$ correspond to those of $\mu$. Furthermore, if $\Phi$ is nonnegative, then $\forall A \in \mathscr{L}, \mu(A)=\inf \Phi(f)$ where inf is taken over all $f$ in $C_{b}(\mathscr{L})$ such that $\chi_{A} \leq f \leq 1$, where $\chi_{A}$ is the characteristic function of $A$. 


\section{Alexandrov lattices}

We can topologize $\operatorname{MR}(\mathscr{L})$ with the weak $(*)$ topology as follows: If $\mu_{\alpha} \in$ $\operatorname{MR}(\mathscr{L})$ then $\mu_{\alpha}$ converges to $\mu$ in the weak topology if and only if $\int f d \mu_{\alpha}$ converges to $\int f d \mu, \forall f \in C_{b}(\mathscr{L})$. In other words, we write

$$
\mu_{\alpha} \in \operatorname{MR}(\mathscr{L}), \quad \mu_{\alpha} \stackrel{w}{\rightarrow} \mu \Leftrightarrow \int f d \mu_{\alpha} \rightarrow \int f d \mu \text { for all } f \in C_{b}(\mathscr{L}) .
$$

Let $\mathscr{L}$ be $\delta$ normal and complement generated (completely normal) lattice of subsets of $X$. Then, Alexandrov's Fundamental Theorem [1b, 4] states that $\operatorname{MR}(\sigma, \mathscr{L})$ is weakly sequentially closed in $\operatorname{MR}(\mathscr{L})$, i.e., if $\mu_{n} \in \operatorname{MR}(\sigma, \mathscr{L})$ and $\mu_{n} \stackrel{w}{\rightarrow} \mu$, then $\mu \in \operatorname{MR}(\sigma, \mathscr{L})$.

Definition. A $\delta$ normal lattice $\mathscr{L}$ of subsets of $X$ is said to be an Alexandrov lattice if $\mu_{n} \in \operatorname{MR}(\sigma, \mathscr{L})$ and $\mu_{n} \stackrel{w}{\rightarrow} \mu$, where $\mu \in \operatorname{MR}(\mathscr{L})$, imply $\mu \in$ $\operatorname{MR}(\sigma, \mathscr{L})$.

Proposition 3.1. Let $\mathscr{L}_{1}$ and $\mathscr{L}_{2}, \mathscr{L}_{1} \subset \mathscr{L}_{2}$, be $\delta$ normal lattices. If $\mathscr{L}_{2}$ is $\mathscr{L}_{1}$ c.p. or c.b. and $\mathscr{L}_{1}$ s.s. $\mathscr{L}_{2}$, then $\mathscr{L}_{1}$ Alexandrov implies that $\mathscr{L}_{2}$ is Alexandrov.

Proof. Suppose $\nu_{n} \in \operatorname{MR}\left(\sigma, \mathscr{L}_{2}\right)$ and $\nu_{n} \stackrel{w}{\rightarrow} \nu, \nu \in \operatorname{MR}\left(\mathscr{L}_{2}\right)$. Since $\mathscr{L}_{1}$ s.s. $\mathscr{L}_{2}$ and $C_{b}\left(\mathscr{L}_{2}\right) \supset C_{b}\left(\mathscr{L}_{1}\right)$, then we have

$$
\mu_{n} \stackrel{w}{\rightarrow} \mu, \quad \mu_{n}=\nu_{n}\left|\in \operatorname{MR}\left(\sigma, \mathscr{L}_{1}\right), \quad \mu=\nu\right| .
$$

Since $\mathscr{L}_{1}$ is Alexandrov, $\mu \in \operatorname{MR}\left(\sigma, \mathscr{L}_{1}\right)$ and, consequently, $\nu \in \operatorname{MR}\left(\sigma, \mathscr{L}_{2}\right)$ (since $\mathscr{L}_{2}$ is $\mathscr{L}_{1}$ c.p. or c.b.). Thus $\mathscr{L}_{2}$ is also an Alexandrov lattice.

Remark. If instead of $\mathscr{L}_{1}$ s.s. $\mathscr{L}_{2}$ we assume that $\mathscr{L}_{1}$ is $\delta$ and $\sigma\left(\mathscr{L}_{1}\right) \subset s\left(\mathscr{L}_{1}\right)$, then in this case $\mu_{n}=\nu_{n} \mid$ is countable, additive, and, therefore, by Choquet's capacity theorem [5], $\mu_{n} \in \operatorname{MR}\left(\sigma, \mathscr{L}_{1}\right)$. Also, $\mu_{n} \stackrel{w}{\rightarrow} \mu=\nu \mid \in M\left(\mathscr{L}_{1}\right)$, but $\mu \leq$ $\rho$ on $\mathscr{L}_{1}$, where $\rho \in \operatorname{MR}\left(\mathscr{L}_{1}\right)$ and $\mu(X)=\rho(X)$, and since $\int f d \mu=\int f d \rho$ for all $f \in C_{b}\left(\mathscr{L}_{1}\right), \mu_{n} \stackrel{w}{\rightarrow} \rho$. Hence $\rho \in \operatorname{MR}\left(\sigma, \mathscr{L}_{1}\right)$ since $\mathscr{L}_{1}$ is Alexandrov and consequently $\mu \in M\left(\sigma, \mathscr{L}_{1}\right)$, and if $\mathscr{L}_{2}$ is $\mathscr{L}_{1}$ c.b. then $\nu \in \operatorname{MR}\left(\sigma, \mathscr{L}_{2}\right)$ and $\mathscr{L}_{2}$ is Alexandrov.

Note that if $\mathscr{L}_{1}$ and $\mathscr{L}_{2}$ are $\delta$ normal and $C\left(\mathscr{L}_{1}\right)=C\left(\mathscr{L}_{2}\right)$, which implies that $\mathscr{L}_{1}$ separates $\mathscr{L}_{2}$, and if $\mathscr{L}_{2}$ is c.p., then $\mathscr{L}_{2}$ is $\mathscr{L}_{1}$ c.p. and $\mu \in \operatorname{MR}\left(\sigma, \mathscr{L}_{1}\right)$. Then by Theorem $2.1 \mu$ extends uniquely to $\nu \in \operatorname{MR}\left(\sigma, \mathscr{L}_{2}\right)$. In other words, we have

Corollary 3.1. Let $\mathscr{L}_{1}$ and $\mathscr{L}_{2}$ be $\delta$ normal, $C\left(\mathscr{L}_{2}\right)=C\left(\mathscr{L}_{1}\right)$, and $\mathscr{L}_{2}$ be c.p. Then $\mathscr{L}_{1}$ Alexandrov implies that $\mathscr{L}_{2}$ is Alexandrov.

By Proposition 3.1, we also have

Corollary 3.2. If $\mathscr{L}$ is $\delta$ normal and c.p., then $\mathscr{L}$ is $\mathscr{Z}(\mathscr{L})$ c.p. and $\mathscr{L}$ is also Alexandrov since $\mathscr{Z}(\mathscr{L})$ is Alexandrov.

Suppose $\mathscr{L}_{1}$ and $\mathscr{L}_{2}$ and $\delta$ normal lattices, $\mathscr{L}_{1} \subset \mathscr{L}_{2}$, and $C\left(\mathscr{L}_{1}\right)=C\left(\mathscr{L}_{2}\right)$. Let $\mu_{n} \stackrel{w}{\rightarrow} \mu$ where $\mu_{n} \in \operatorname{MR}\left(\sigma, \mathscr{L}_{1}\right)$ and $\mu \in \operatorname{MR}\left(\mathscr{L}_{1}\right)$. Then, if $\nu_{n} \in$ $\operatorname{MR}\left(\sigma, \mathscr{L}_{2}\right)$ is the unique extension of $\mu_{n}$, and $\nu$ is that of $\mu$, we have $\nu_{n} \stackrel{w}{\rightarrow} \nu$ and $\nu \in \operatorname{MR}\left(\sigma, \mathscr{L}_{2}\right)$ assuming $\mathscr{L}_{2}$ is Alexandrov. Therefore, $\mu \in \operatorname{MR}\left(\sigma, \mathscr{L}_{1}\right)$ and $\mathscr{L}_{1}$ is Alexandrov. This fact together with Corollary 3.1 gives 
Corollary 3.3. If $\mathscr{L}_{1}$ and $\mathscr{L}_{2}$ are $\delta$ normal lattices, $\mathscr{L}_{2}$ is c.p. or c.b., and $C\left(\mathscr{L}_{2}\right)=C\left(\mathscr{L}_{1}\right)$, then $\mathscr{L}_{1}$ is Alexandrov if and only if $\mathscr{L}_{2}$ is Alexandrov.

Proposition 3.2. If $\mathscr{L}$ is a $\delta$ normal and c.p. lattice of subsets of $X$ and $\mu_{n} \stackrel{w}{\rightarrow} \mu$ where $\mu_{n} \in M(\sigma, \mathscr{L}), \mu \in M(\mathscr{L})$, then $\mu \in M(\sigma, \mathscr{L})$.

Proof. Let $\mu_{n} \in M(\sigma, \mathscr{L})$ and $\mu_{n} \stackrel{w}{\rightarrow} \mu$. The functional $\Phi_{n}(f)=\int f d \mu_{n}$ is a bounded linear functional of $f \in C_{b}(\mathscr{L})$ and, by A.R.T., we have $\Phi_{n}(f)=$ $\int f d \mu_{n}=\int f d \nu_{n}, \nu_{n} \in \operatorname{MR}(\mathscr{L}), \mu_{n}(X)=\nu_{n}(X)$, and $\mu_{n} \leq \nu_{n}$ on $\mathscr{L}$. Since $\mathscr{L}$ is normal and c.p., we also have $\nu_{n} \in \operatorname{MR}(\sigma, \mathscr{L})$. Thus $\int f d \nu_{n}=$ $\int f d \mu_{n} \rightarrow \int f d \mu$. Also, by A.R.T., $\Phi(f)=\int f d \mu=\int f d \nu, \nu \in \operatorname{MR}(\mathscr{L})$ and $\mu(X)=\nu(X)$ on $\mathscr{L}$. Therefore, $\int f d \nu_{n} \rightarrow \int f d \nu$ or $\nu_{n} \stackrel{w}{\rightarrow} \nu$. Since $\mathscr{L}$ is $\delta$ normal and c.p., by Corollary 3.2, $\nu \in \operatorname{MR}(\sigma, \mathscr{L})$. On the other hand, we have $\mu \leq \nu$ on $\mathscr{L}$. Therefore, $\mu \in \operatorname{MR}(\sigma, \mathscr{L})$.

Proposition 3.3. Let $\mathscr{L}_{1}$ and $\mathscr{L}_{2}$ be lattices of subsets of $X$. Suppose $\mathscr{L}_{1}$ separates $\mathscr{L}_{2}$ and $\mathscr{L}_{1}$ is an Alexandrov lattice. If $M\left(\sigma, \mathscr{L}_{2}^{\prime}\right) \cap \mathrm{MR}\left(\mathscr{L}_{2}\right) \subset M\left(\sigma, \mathscr{L}_{2}\right)$ then $\mathscr{L}_{2}$ is also an Alexandrov lattice.

Proof. Let $\nu_{n} \stackrel{w}{\rightarrow} \nu$ where $\nu_{n} \in \operatorname{MR}\left(\sigma, \mathscr{L}_{2}\right)$ and $\nu \in \operatorname{MR}\left(\mathscr{L}_{2}\right)$. Since $\mathscr{L}_{1}$ is an Alexandrov lattice and $\nu_{n} \mid=\mu_{n} \in \operatorname{MR}\left(\sigma, \mathscr{L}_{1}\right)$, we have $\nu_{n}\left|=\mu_{n} \stackrel{w}{\rightarrow} \nu\right|=$ $\mu \in \operatorname{MR}\left(\sigma, \mathscr{L}_{1}\right) . \mathscr{L}_{1}$ actually coseparates $\mathscr{L}_{2}$ since $\mathscr{L}_{1}$ separates $\mathscr{L}_{2}$ and $\mathscr{L}_{1}$ is normal. It is not difficult to see that $\nu \in \operatorname{MR}\left(\sigma, \mathscr{L}_{2}\right)$ since $\nu$ must be $\mathscr{L}$-regular on $\mathscr{L}_{2}^{\prime} ;$ namely, $\nu \in \operatorname{MR}\left(\mathscr{L}_{2}\right)$ by hypothesis and $\nu \in M\left(\sigma, \mathscr{L}_{2}^{\prime}\right)$ since $\nu$ is $\mathscr{L}_{1}$-regular on $\mathscr{L}_{2}^{\prime}$. Clearly, if $M\left(\sigma, \mathscr{L}_{2}^{\prime}\right) \cap \operatorname{MR}\left(\mathscr{L}_{2}\right) \subset M\left(\sigma, \mathscr{L}_{2}\right)$ then $\nu \in \operatorname{MR}\left(\sigma, \mathscr{L}_{2}\right)$. Therefore, $\mathscr{L}_{2}$ is an Alexandrov lattice.

Let $\mathscr{L}_{1}$ be a lattice of subsets of $X$ and $\mathscr{L}_{2}$ be a lattice of subsets of $Y$. Again, we assume that $\mathscr{L}_{1}$ and $\mathscr{L}_{2}$ are $\delta$ normal.

Let $T: X \rightarrow Y$ be $\mathscr{L}_{1}-\mathscr{L}_{2}$ continuous. Consider a mapping $A: C_{b}\left(\mathscr{L}_{2}\right) \rightarrow$ $C_{b}\left(\mathscr{L}_{1}\right)$ such that $A$ is linear and bounded.

If the mapping $A$ is defined by $A g=g T$ where $g \in C_{b}\left(\mathscr{L}_{2}\right)$, then define the adjoint map by $A^{\prime}: C_{b}\left(\mathscr{L}_{1}\right)^{\prime} \rightarrow C_{b}\left(\mathscr{L}_{2}\right)^{\prime}$ where $C_{b}\left(\mathscr{L}_{i}\right)^{\prime}$ is congruent to $\operatorname{MR}\left(\mathscr{L}_{i}\right)$ $(i=1,2)$ and $\left(A^{\prime} \Phi\right)(g)=\Phi(A g)$. By A.R.T. we have $\Phi \leftrightarrow \mu, \mu \in \operatorname{MR}\left(\mathscr{L}_{1}\right)$ and $A^{\prime} \Phi \leftrightarrow \nu, \nu \in \operatorname{MR}\left(\mathscr{L}_{2}\right)$. Then, $\Phi(A g)=\int A g d \mu$ and $\left(A^{\prime} \Phi\right)(g)=\int g d \nu$ for all $g \in C_{b}\left(\mathscr{L}_{2}\right)$ and consequently $A^{\prime}: \operatorname{MR}\left(\mathscr{L}_{1}\right) \rightarrow \operatorname{MR}\left(\mathscr{L}_{2}\right)$ where $A^{\prime} \mu=\nu$ and $\int_{Y} g d \nu=\left(A^{\prime} \Phi\right)(g)=\Phi(A g)=\int_{X} A g d \mu=\int_{X} g T d \mu=\int_{Y} g d \mu T^{-1}$, $g \in C_{b}\left(\mathscr{L}_{2}\right)$.

Note that $A$ is a linear mapping and that $A g_{1} g_{2}=g_{1} T g_{2} T=A g_{1} A g_{2}$. Therefore, $A$ is an algebra homomorphism. Also, we have $\|A g\|=\|g T\| \leq$ $\|g\|$. Indeed, $A$ is bounded. If $T$ is surjective then $\|A g\|=\|g\|$, i.e., $A$ is an isometry, and consequently $A$ is invertible.

Some basic properties of $A^{\prime}$ are collected in the following.

Proposition 3.4. (a) If $\mu \geq 0$ then $\nu=A^{\prime} \mu \geq 0$.

(b) $A^{\prime} \mu=\nu \geq \mu T^{-1}$ on $\mathscr{L}_{2}$ and $\nu(Y)=\mu T^{-1}(Y)$.

(c) $A^{\prime}\left(I R\left(\mathscr{L}_{1}\right)\right) \subset \operatorname{IR}\left(\mathscr{L}_{2}\right)$.

(d) $\left.A^{\prime}\right|_{I R\left(\mathscr{L}_{1}\right)}$ is weakly continuous.

Proof. We show only (b). Further details can be found in [2]. We have $\mu T^{-1}(L)=\int_{L} d \mu T^{-1}=\int_{Y} \chi_{L} d \mu T^{-1} \leq \int_{Y} g d \mu T^{-1}=\int_{Y} g d \nu$, where $g \in$ 
$C_{b}\left(\mathscr{L}_{2}\right)$ and $\chi_{L} \leq g \leq 1$. Therefore, $\mu T^{-1}(L) \leq \nu(L)$ for all $L \in \mathscr{L}_{2}$. If $g=1$, we obtain $\int_{Y} d \nu=\int_{Y} d \mu T^{-1}$. Hence $\nu(Y)=\mu T^{-1}(Y)$.

Proposition 3.5. (a) If $\mathscr{L}_{2}$ is c.p., then $A^{\prime}\left(\operatorname{MR}\left(\sigma, \mathscr{L}_{1}\right)\right) \subset \operatorname{MR}\left(\sigma, \mathscr{L}_{2}\right)$.

(b) If $T$ is surjective and $\mathscr{L}_{1}$ is $T^{-1}\left(\mathscr{L}_{2}\right) \quad$ c.b., then $\operatorname{MR}\left(\sigma, \mathscr{L}_{2}\right) \subset$ $A^{\prime}\left(\operatorname{MR}\left(\sigma, \mathscr{L}_{1}\right)\right)$.

(c) If (a) and (b) hold then, $A^{\prime}\left(\operatorname{MR}\left(\sigma, \mathscr{L}_{1}\right)\right)=\operatorname{MR}\left(\sigma, \mathscr{L}_{2}\right)$.

Proof. Here we show only (a). Suppose $\mathscr{L}_{2}$ is c.p. Let $\mu \in \operatorname{MR}\left(\sigma, \mathscr{L}_{1}\right)$ and consider any element of $A^{\prime}\left(\operatorname{MR}\left(\sigma, \mathscr{L}_{1}\right)\right), A^{\prime} \mu$. We must show that $A^{\prime} \mu=\nu \in$ $\operatorname{MR}\left(\sigma, \mathscr{L}_{2}\right)$. By A.R.T. we have $\mu \leftrightarrow \Phi$ and $\Phi$ is $\sigma$-smooth. In fact, consider $\left\{g_{n}\right\}, g_{n} \in C_{b}\left(\mathscr{L}_{2}\right), g_{n} \downarrow 0$. Then $g_{n} T \downarrow 0$ and, therefore, $\lim \int g_{n} T d \mu=$ 0 . However, $\lim \int g_{n} T d \mu=\lim \int g_{n} d \nu=0$, which means $\Phi \leftrightarrow \nu$ where $\Phi(g)=\int d d \nu$ for all $g \in C_{b}\left(\mathscr{L}_{2}\right)$. Since $\Phi$ is $\sigma$-smooth and $\mathscr{L}_{2}$ is c.p., we have $A^{\prime} \mu=\nu \in \operatorname{MR}\left(\sigma, \mathscr{L}_{2}\right)$. Hence $A^{\prime}\left(\operatorname{MR}\left(\sigma, \mathscr{L}_{1}\right)\right) \subset \operatorname{MR}\left(\sigma, \mathscr{L}_{2}\right)$.

Proposition 3.6. (1) Under the assumption Proposition 3.5 (a), if $\mathscr{L}_{1}$ is an Alexandrov lattice and

$$
\mu_{n} \stackrel{w}{\rightarrow} \mu, \quad \mu_{n} \in \operatorname{MR}\left(\sigma, \mathscr{L}_{1}\right),
$$

then $A^{\prime} \mu_{n} \stackrel{w}{\rightarrow} A^{\prime} \mu$ and $A^{\prime} \mu_{n}, A^{\prime} \mu \in \operatorname{MR}\left(\sigma, \mathscr{L}_{2}\right)$.

(2) Under the assumptions Proposition 3.5 (a), (b) and if $A$ is surjective, then $\mathscr{L}_{1}$ Alexandrov implies that $\mathscr{L}_{2}$ is Alexandrov.

Proof. (1) Since $\mathscr{L}_{1}$ is Alexandrov, we have $\mu_{n} \stackrel{w}{\rightarrow} \mu, \mu \in \operatorname{MR}\left(\sigma, \mathscr{L}_{1}\right)$. Then, by Proposition 3.4(d), $A^{\prime} \mu_{n} \rightarrow A^{\prime} \mu$. By Proposition 3.5(a), $A^{\prime} \mu_{n} \in \operatorname{MR}\left(\sigma, \mathscr{L}_{2}\right)$ and $A^{\prime} \mu \in A^{\prime}\left(\operatorname{MR}\left(\sigma, \mathscr{L}_{1}\right)\right) \subset \operatorname{MR}\left(\sigma, \mathscr{L}_{2}\right)$.

(2) Let $\nu_{n} \in \operatorname{MR}\left(\sigma, \mathscr{L}_{2}\right)$. Then $\nu_{n} \stackrel{w}{\rightarrow} \nu \in \operatorname{MR}\left(\mathscr{L}_{2}\right)$. By Proposition 3.5(b), we have $\nu_{n}=A^{\prime} \mu_{n}, \mu_{n} \in \operatorname{MR}\left(\sigma, \mathscr{L}_{1}\right)$ and $\nu=A^{\prime} \mu, \mu \in \operatorname{MR}\left(\mathscr{L}_{1}\right)$. Since $A$ is surjective, we have $\int A g d \mu_{n}=\int g d \nu_{n} \rightarrow \int g d \nu=\int A g d \mu$. Hence $\mu_{n} \stackrel{w}{\rightarrow} \mu$. Since $\mathscr{L}_{1}$ is Alexandrov, $\mu \in \operatorname{MR}\left(\sigma, \mathscr{L}_{1}\right)$. Therefore, by Proposition 3.5(a), $A^{\prime} \mu=\nu \in \operatorname{MR}\left(\sigma, \mathscr{L}_{2}\right)$. Hence $\mathscr{L}_{2}$ is Alexandrov.

Thus, under the above assumptions, the measure defined on Alexandrov lattices is invariant under adjoint mappings.

\section{REFERENCES}

1. A. D. Alexandrov, Additive set-functions in abstract spaces, Mat. Sb. (N.S.) (a) 8(50) (1940), 307-348; (b) 9(51) (1941), 563-628; (c) 13 (55) (1943), 169-238.

2. G. Bachman and P. Stratigos, Some applications of the adjoint to lattice regular measures, Ann. Mat. Pura Appl. (4) 138 (1984), 379-397.

3. G. Bachman and A. Sultan, On regular extensions of measures, Pacific J. Math. vol. 86, No. 2 (1980), 389-395.

4. H. Bergstrom, Weak convergence of measures, Academic Press, NY and London, 1982.

5. G. Choquet, theory of capacities, Ann. Inst. Fourier (Grenoble) 5 (1953-1954), 131-295.

6. V. S. Varadarajan, Measures on topological spaces, Amer. Math. Soc. Transl., vol. 48, Amer. Math. Soc., Providence, RI, 1965, pp. 161-228.

Department of Mathematics and Physics, New York Institute of Technology, Old WeSTBURY, NeW YORK, 11568 